STRUCTURAL BIOLOGY COMMUNICATIONS

ISSN 2053-230X

Received 11 May 2021

Accepted 16 July 2021

Edited by M. W. Bowler, European Molecular Biology Laboratory, France

Keywords: $\beta$-barrels; ligand-binding sites; conformational change; virulence factors; virulence-associated proteins; Rhodococcus equi.

PDB reference: VapB from the intracellular pathogen Rhodococcus equi, $7 \mathrm{~b} 1 \mathrm{z}$

Supporting information: this article has supporting information at journals.iucr.org/f

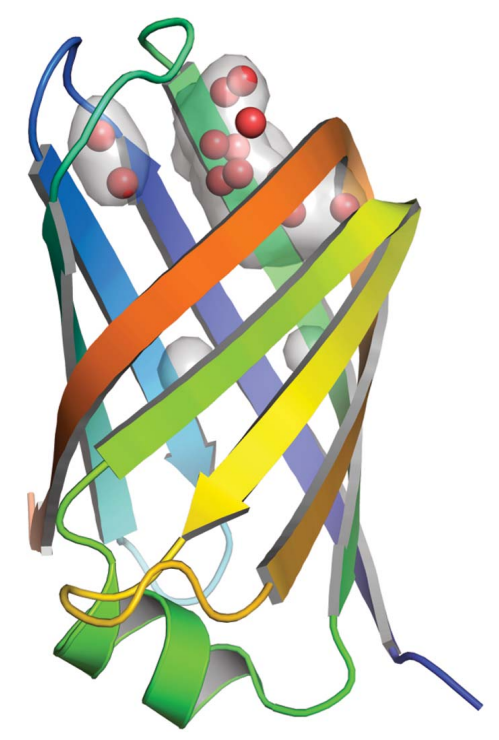
OPEN $\odot$ ACCESS

\section{Conformational changes of loops highlight a potential binding site in Rhodococcus equi VapB}

\author{
Christina Geerds, ${ }^{a}$ Albert Haas ${ }^{\mathrm{b}}$ and Hartmut H. Niemann ${ }^{\mathrm{a} *}$ \\ ${ }^{\mathbf{a}}$ Department of Chemistry, Bielefeld University, Universitaetsstrasse 25, 33615 Bielefeld, Germany, and ${ }^{\mathbf{b}}$ Institute for Cell \\ Biology, University of Bonn, Ulrich-Haberland-Strasse 61a, 53121 Bonn, Germany. *Correspondence e-mail: \\ hartmut.niemann@uni-bielefeld.de
}

Virulence-associated proteins (Vaps) contribute to the virulence of the pathogen Rhodococcus equi, but their mode of action has remained elusive. All Vaps share a conserved core of about 105 amino acids that folds into a compact eight-stranded antiparallel $\beta$-barrel with a unique topology. At the top of the barrel, four loops connect the eight $\beta$-strands. Previous Vap structures did not show concave surfaces that might serve as a ligand-binding site. Here, the structure of VapB in a new crystal form was determined at $1.71 \AA$ resolution. The asymmetric unit contains two molecules. In one of them, the loop regions at the top of the barrel adopt a different conformation from other Vap structures. An outward movement of the loops results in the formation of a hydrophobic cavity that might act as a ligand-binding site. This lends further support to the hypothesis that the structural similarity between Vaps and avidins suggests a potential binding function for Vaps.

\section{Introduction}

Rhodococcus equi is a Gram-positive environmental soil bacterium that can cause severe bronchopneumonia in young foals (Vázquez-Boland \& Meijer, 2019). It also poses a threat to immunocompromised humans such as AIDS patients. $R$. equi infects lung alveolar macrophages, where it multiplies in a remodelled compartment, the Rhodococcus-containing vacuole (Zink et al., 1987; Fernandez-Mora et al., 2005). This is an unusual phagolysosome that maintains a near-neutral $\mathrm{pH}$ (von Bargen et al., 2019). A virulence-associated plasmid of some $85 \mathrm{kbp}$ is absolutely required for infection of foals (Takai et al., 1991; Tkachuk-Saad \& Prescott, 1991) and encodes a key factor for infection and intracellular multiplication: virulenceassociated protein A (VapA; Jain et al., 2003). VapA is a surface-attached $17 \mathrm{kDa}$ protein that is released into the phagosome during infection and that increases the permeability of phagosome and lysosome membranes to some ions and protons (von Bargen et al., 2019). The critical activity of VapA is the collapse of the $\mathrm{pH}$ gradient across the phagosome membrane, as chemical $\mathrm{pH}$ neutralization of the endocytic and phagocytic continuum using any of several $\mathrm{pH}$-neutralizing drugs enables virulence-plasmid-deficient (avirulent) R. equi to multiply in macrophages. Interestingly, a proteinase $\mathrm{K}$ digested version of VapA, which corresponds to the VapB fragment in this study, still has membrane-permeabilizing activity and supports intracellular multiplication of plasmidless R. equi (von Bargen et al., 2019).

Although the membrane-binding and permeabilizing activities of VapA have been clearly documented (Wright $e t$ al., 2018; von Bargen et al., 2019), it is not known how VapA exerts its effect on membranes. The same applies to virulenceassociated protein B (VapB), which is also encoded on a 
virulence plasmid; this plasmid is mostly absent from foal isolates and is predominantly found in porcine $R$. equi isolates (Ribeiro et al., 2011; Letek et al., 2008). Recent data suggest that VapB, although 78\% identical to VapA in its amino-acid sequence, is not required for the virulence of VapB-producing $R$. equi strains (Willingham-Lane et al., 2018). It has been reported that VapB binds more weakly to yeast plasma membranes and to some liposomes than VapA does (Wright et al., 2018). However, VapB does bind well to some membranes (A. Haas. P. Hansen \& J. Kniewel, unpublished data), possibly depending on their precise composition.

We reasoned that in the absence of a VapA structure and given the very high level of sequence identity $(78 \%)$ of VapB to VapA, solving the structure of VapB could hint at possible membrane-interacting surfaces, which then could provide clues as to how VapA exerts its pathogenic effects (Geerds et al., 2014). Shortly after our VapB structure, the very similar structures of R. equi VapD and VapG were published (Whittingham et al., 2014; Okoko et al., 2015). Vap proteins consist of an N-terminal region that is variable in sequence and is probably intrinsically unstructured and a stably folded C-terminal core with high sequence conservation. The protease-resistant core of $R$. equi Vap proteins forms an eightstranded $\beta$-barrel consisting of two Greek-key motifs. Vaps have internal symmetry, with a pseudo-twofold axis relating the two Greek-key motifs, suggesting that the structure arose by gene duplication (Whittingham et al., 2014). A short helix connects the two half-barrels, resulting in an unusual topology that is very different from the antiparallel all-next-neighbour topology typically found in (eight-stranded) $\beta$-barrels. We term the end of the barrel containing the $\alpha$-helix and both termini the bottom. The top end contains the four connections between odd- and even-numbered strands, two of which are next neighbours $(\beta 1-\beta 2$ and $\beta 5-\beta 6)$ and the other two of which are crossover connections $(\beta 3-\beta 4$ and $\beta 7-\beta 8)$.

The Vap surface contains a flat region devoid of side chains due to the accumulation of glycines. In the VapD structure, two octyl- $\beta$-D-glucoside molecules from the crystallization solution bind to this 'bald' spot that may allow R. equi Vaps to interact with large nonpolar surfaces (Whittingham et al., 2014). We had suggested a functional similarity between Vaps and avidins, which also form eight-stranded $\beta$-barrels (Geerds et al., 2014). However, avidins have a different, all-nextneighbour topology. Avidins bind biotin in a groove at the top of the molecule (Livnah et al., 1993; Weber et al., 1989). Whittingham and coworkers also noted the structural similarity between VapD and bradavidin 2, an avidin-like protein from Bradyrhizobium japonicum (Leppiniemi et al., 2013), but suggested that VapD is not involved in small-molecule binding due to the absence of a cavity (Whittingham et al., 2014). Okoko and coworkers found no significant depressions on the surfaces of VapB, VapD and VapG that would indicate the presence of a ligand-binding cavity or an enzyme active site (Okoko et al., 2015). Moreover, Okoko and coworkers noted that avidins lack crossover strands due to their antiparallel allnext-neighbour topology. As a result, the avidin barrels have intrinsically more open structures that allow one end of the barrel to develop into a ligand-binding cavity, in which the barrel interior forms the base. In contrast, Vap proteins have less potential for a binding site due to the crossover connections linking strands $\beta 3$ to $\beta 4$ and $\beta 7$ to $\beta 8$ at the top and the $\alpha$-helix that seals the bottom (Okoko et al., 2015). Nevertheless, experimental data show that $R$. equi Vaps can interact with component(s) of the host cell, most notably membranes. Here, we present a new structure of VapB that highlights a potential binding site for a putative host cell-derived ligand.

\section{Materials and methods}

\subsection{Macromolecule production}

VapB was expressed and purified as described by Geerds $e t$ al. (2014), with some differences in the digestion with proteinase K. $50 \mathrm{mg}$ VapB was digested with proteinase K (Roth, product key 7528.1) at a $25: 1$ protein:protease ratio in $50 \mathrm{ml}$ phosphate-buffered saline for $4 \mathrm{~h}$ at $37^{\circ} \mathrm{C}$. The protease was inactivated by adding phenylmethylsulfonyl fluoride (PMSF) to a final concentration of $2 \mathrm{~m} M$ and incubating on ice for $30 \mathrm{~min}$. The solution was then dialyzed against $2125 \mathrm{mM}$ Tris $\mathrm{pH} 7,20 \mathrm{~m} M \mathrm{NaCl}, 0.1 \mathrm{~m} M$ PMSF overnight, with a protein recovery of about $50 \%(25.4 \mathrm{mg})$. The proteinase $\mathrm{K}$ digest resulted in a single VapB band on SDS-PAGE, in contrast to a previous proteinase $\mathrm{K}$ digest, where a double band was observed (Geerds et al., 2014). Proteinase K-digested VapB was further purified by anion-exchange chromatography on Source Q (GE Healthcare) resin equilibrated in $25 \mathrm{mM}$ Tris pH 7, $20 \mathrm{mM} \mathrm{NaCl}$. Elution with a salt gradient up to $1 M$ $\mathrm{NaCl}$ resulted in a broad and nonsymmetric peak, indicating some heterogeneity. Late peak fractions were pooled, dialyzed against $25 \mathrm{~m} M$ Tris pH 7.0, $20 \mathrm{~m} M \mathrm{NaCl}$ overnight, concentrated to $16.8 \mathrm{mg} \mathrm{ml}^{-1}$ using Vivaspin concentrators, frozen in small aliquots and stored at $-80^{\circ} \mathrm{C}$. The recovery after proteinase $\mathrm{K}$ digestion and purification was about $5 \%$ $(2.4 \mathrm{mg})$. Macromolecule-production information is summarized in Table 1.

\subsection{Crystallization}

Initial crystals grew in condition F4 of The JCSG Core I Suite (20\% PEG 3350, 0.2 M magnesium nitrate). The crystal used for data collection grew in a sitting-drop vapour-diffusion setup under optimized conditions (14\% PEG 4000, $0.2 \mathrm{M}$ magnesium nitrate) with $10 \mathrm{mg} \mathrm{ml}^{-1}$ protein in $25 \mathrm{~m} M$ Tris $\mathrm{pH}$ $7,0,20 \mathrm{mM} \mathrm{NaCl}$ at $20^{\circ} \mathrm{C}$ using $1 \mu \mathrm{l}$ protein solution plus $0.5 \mu \mathrm{l}$ reservoir solution. A drop ratio of 2:1 protein:precipitant solution has been shown to yield more crystallization hits than a ratio of $1: 1$ or $1: 2(\mathrm{Ng}$ et al., 2016), and we routinely use 2:1 drops (Niemann et al., 2006; Thoms et al., 2011; Schreiner \& Niemann, 2012; Barden et al., 2014; Moritzer \& Niemann, 2019; Meyer et al., 2020). The crystal grew to final dimensions of about $120 \times 70 \mu \mathrm{m}$ in about three weeks. The crystal was cryoprotected in 18\% PEG 4000, $0.2 M$ magnesium nitrate, $20 \%$ glycerol and flash-cooled in liquid nitrogen. Crystallization information is summarized in Table 2. 
Table 1

Macromolecule-production information.

Source organism

DNA source

Forward primer $\dagger$

Reverse primer $\ddagger$

Expression vector

Expression host

Complete amino-acid sequence of the construct produced $\S$
Rhodococcus equi strain PAM 1593

Sequenced virulence plasmid

CAT CAT CAC CAC CAT CAC GTG CTG GAT TCC GGA GGC GGC (His His His His His His Val36 Leu37 Asp38 Ser39 Gly Gly Gly)

GTG GC|G GCC GCT CTA TTA TTA TGC AAC CTC CCA GTT GTG (His Asn Trp Glu Val Ala197 Stop Stop)

pETite N-His Kan (BioCat, Heidelberg, Germany)

E. coli BL21 (DE3)

MHHHHHHVLDSGGGSALLKDGAGSGEVGSQ AYDSSTVSSNLQKAETNGPVGLAGTAEQ EQQYDVHGNVISAAVYQKFHVYGPEDMV FDGDAGGLTIPGAGAFWGTLFTSDLQRI YKDTVSFQYNALGTYLNINFFDSSGGFI GHIQAGAVSAVVGVGGGSGSWHNWEVA

† The codons for the hexahistidine tag are in bold and the sequence coding for VapB starting at Val36 is in italics. $\ddagger$ The sequence coding for VapB is in italics, two stop codons are in bold, the NotI restriction site is underlined and the cleavage site of NotI is indicated by a vertical line. $\S$ The expressed construct comprises residues Val36Ala197 of VapB. The additional hexahistidine tag is shown in bold.

Table 2

Crystallization.

\begin{tabular}{ll}
\hline Method & Vapour diffusion \\
Plate type & Cryschem M 24-well sitting drop \\
Temperature $(\mathrm{K})$ & 293 \\
Protein concentration $\left(\mathrm{mg} \mathrm{ml}^{-1}\right)$ & 10 \\
Buffer composition of protein solution & $25 \mathrm{mM}$ Tris pH 7.0, 20 $\mathrm{mM} \mathrm{NaCl}$ \\
Composition of reservoir solution & $14 \% \mathrm{PEG} 4000,0.2 \mathrm{M} \mathrm{Mg}\left(\mathrm{NO}_{3}\right)_{2}$ \\
Volume and ratio of drop & $1 \mu \mathrm{l}$ protein solution $+0.5 \mu \mathrm{l}$ \\
& reservoir solution \\
Volume of reservoir $(\mu \mathrm{l})$ & 500 \\
\hline
\end{tabular}

\subsection{Data collection and processing}

Data were collected on beamline P14 operated by EMBL Hamburg at the PETRA III storage ring, DESY, Hamburg, Germany during setup of this beamline (on 5 October 2012) with an unfocused beam, an aperture size of $150 \mu \mathrm{m}$ and a fixed wavelength of $1.23953 \AA$. The beamline was equipped with a vertical 'hanging' spindle and a PILATUS 6M detector. Data were collected in two runs. After a short sweep (316 images), the detector distance was decreased to achieve higher resolution. Data were indexed and integrated with $X D S$ (Kabsch, 2010) and both sweeps were scaled together with $X S C A L E$ using zero-dose extrapolation (Diederichs et al., 2003). Data-collection and processing statistics are summarized in Table 3.

\subsection{Structure solution and refinement}

The structure was solved by molecular replacement in Phaser (McCoy et al., 2007) using a preliminary model of the published $P 6_{1} 22$ structure (Geerds et al., 2014) as a search model. The preliminary $P 6_{1} 22$ structure had been refined against $1.9 \AA$ resolution home-source data before the highresolution synchrotron data for the $P 6_{1} 22$ crystal form became available. Two copies of VapB could easily be located and the structure was manually rebuilt in Coot (Casañal et al., 2020) and refined in REFMAC5 (Murshudov et al., 2011) to facilitate
Table 3

Data collection and processing.

Values in parentheses are for the highest resolution shell.

\begin{tabular}{ll}
\hline Diffraction source & $\begin{array}{l}\text { EMBL beamline P14 (unfocused beam) } \\
\text { PETRA III, DESY }\end{array}$ \\
Wavelength $(\AA)$ & 1.23953 \\
Temperature $(\mathrm{K})$ & 100 \\
Detector & PILATUS 6M \\
Crystal-to-detector distance $(\mathrm{mm})$ & First sweep, 290; second sweep, 243 \\
Rotation range per image $\left({ }^{\circ}\right)$ & 0.1 \\
Total rotation range $\left({ }^{\circ}\right)$ & First sweep, 31.6; second sweep, 180.4 \\
Exposure time per image $(\mathrm{s})$ & 1 \\
Space group & $C 222_{1}$ \\
$a, b, c(\AA)$ & $56.94,65.34,124.26$ \\
Mosaicity $\left({ }^{\circ}\right)$ & 0.12 \\
Resolution range $(\AA)$ & $50-1.71(1.75-1.71)$ \\
Total No. of reflections & $178471(8435)$ \\
No. of unique reflections & $25467(1845)$ \\
Completeness $(\%)$ & $99.8(99.3)$ \\
Multiplicity & $7.0(4.6)$ \\
$\langle I / \sigma(I)\rangle$ & $28.7(2.8)$ \\
$C C_{1 / 2}(\%)$ & $100(85.0)$ \\
$R_{\text {meas }}(\%)$ & $3.9(65.7)$ \\
Overall $B$ factor from Wilson & 33
\end{tabular}

plot $\left(\AA^{2}\right)$

Table 4

Structure solution and refinement.

Values in parentheses are for the highest resolution shell.

\begin{tabular}{ll}
\hline Resolution range $(\AA)$ & $42.93-1.71(1.78-1.71)$ \\
Completeness $(\%)$ & $99.8(99)$ \\
No. of reflections, working set & $25424(2640)$ \\
No. of reflections, test set & $1265(133)$ \\
Final $R_{\text {work }}$ & $0.1539(0.1816)$ \\
Final $R_{\text {free }}$ & $0.1816(0.2997)$ \\
No. of non-H atoms & \\
$\quad$ Protein & 1733 \\
Water & 184 \\
Other (nitrate, glycerol) & 38 \\
Total & 1955 \\
R.m.s. deviations & \\
Bonds $(\AA)$ & 0.009 \\
$\quad$ Angles $\left({ }^{\circ}\right)$ & 0.910 \\
Average $B$ factors $\left(\AA^{2}\right)$ & \\
Protein & 30.4 \\
$\quad$ Water & 38.4 \\
$\quad$ Other (nitrate, glycerol) & 47.1 \\
Ramachandran plot & \\
$\quad$ Most favoured $(\%)$ & 97.20 \\
Allowed $(\%)$ & 2.80 \\
\hline
\end{tabular}

quick cycles of building and refinement. Final refinements were performed in phenix.refine with TLS refinement enabled (Liebschner et al., 2019), yielding lower $R$ factors. Figures were generated with $P y M O L$. Refinement statistics are summarized in Table 4.

\section{Results and discussion}

Here, we determined the structure of the protease-resistant VapB core in a new crystal form with two molecules in the asymmetric unit. The crystals diffracted to $1.71 \AA$ resolution and the structure was solved by molecular replacement. Electron density is visible for residues Gln86-Trp194, while three additional C-terminal residues were resolved in the published VapB structure (Geerds et al., 2014). Here, we used 
a different batch of protein that may chemically differ from the previously published batch due to differences in the proteinase $\mathrm{K}$ digestion (see Section 2) that resulted in different behaviour of the protein in SDS-PAGE and ionexchange chromatography. The crystallization conditions also differ from the published conditions, which did not reproducibly yield crystals. It is unclear whether the proteinase $\mathrm{K}$ digestion in this work removed the C-terminal residues that were previously visible or whether these residues are present but disordered in the new structure.

The two monomers in the asymmetric unit arrange around a pseudo-twofold axis (rotation of $\sim 176^{\circ}$, translation of $\sim 3 \AA$; Fig. 1a). A nitrate ion from the crystallization cocktail, which contained $200 \mathrm{~m} M$ magnesium nitrate, binds between strands

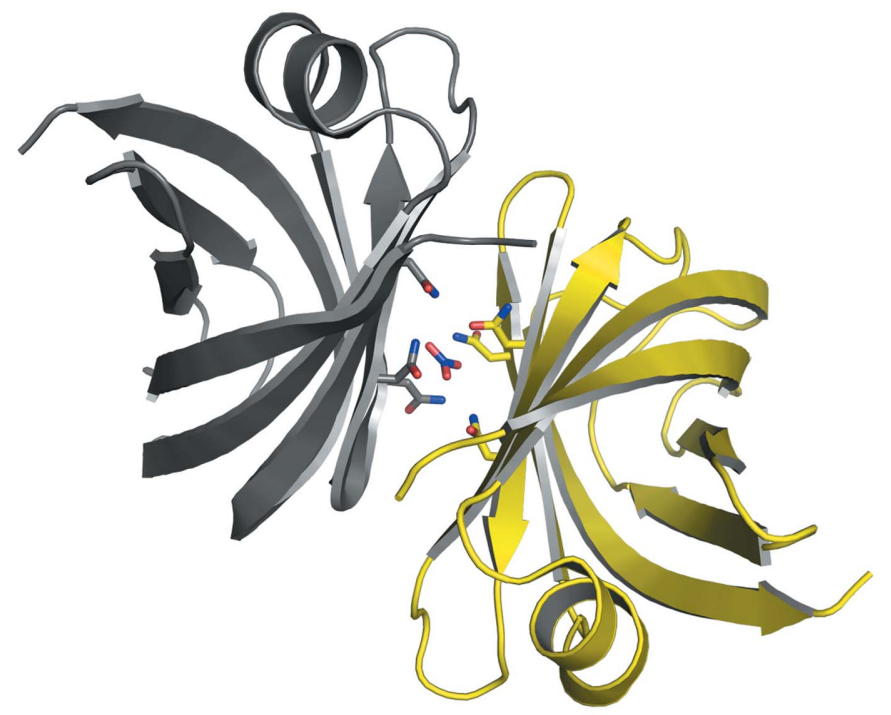

(a)

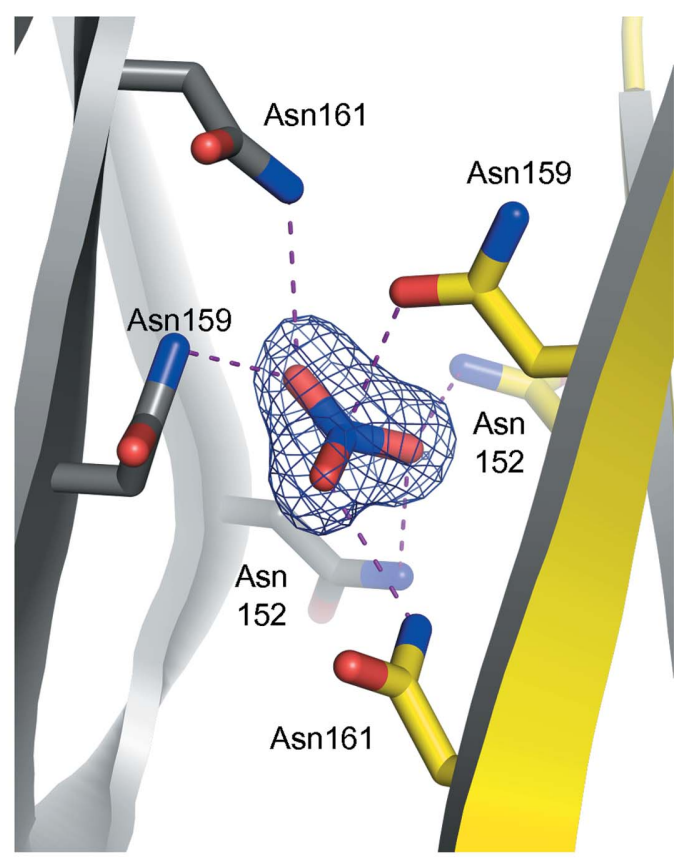

(b)

Figure 1

A highly coordinated nitrate ion from the crystallization cocktail bridges two molecules in the asymmetric unit. (a) View roughly along the pseudotwofold axis. Chain $A$ is depicted in grey and chain $B$ is in yellow. The side chains of Asn152, Asn159 and Asn161 are shown as sticks. (b) Dashed purple lines represent polar contacts between the nitrate ion (residue B201) and Asn152, Asn159 and Asn161 from chains $A$ (grey) and $B$ (yellow). A simulatedannealing OMIT $2 m F_{\mathrm{o}}-D F_{\mathrm{c}}$ map is contoured at $1 \sigma$ around the nitrate ion.

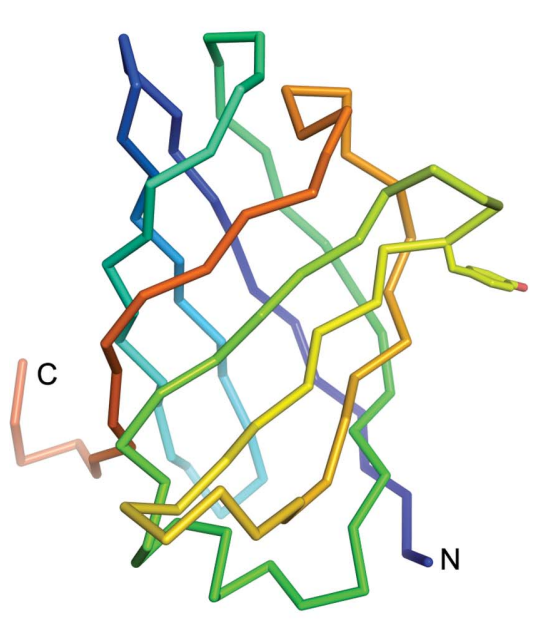

(a)

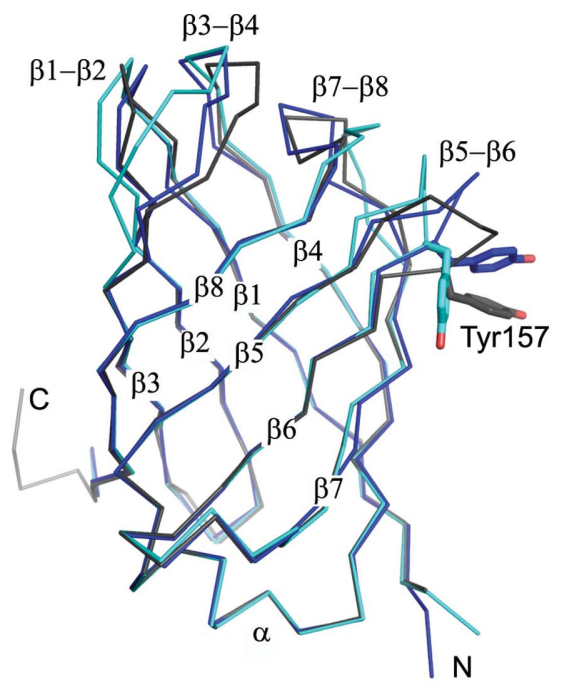

(b)

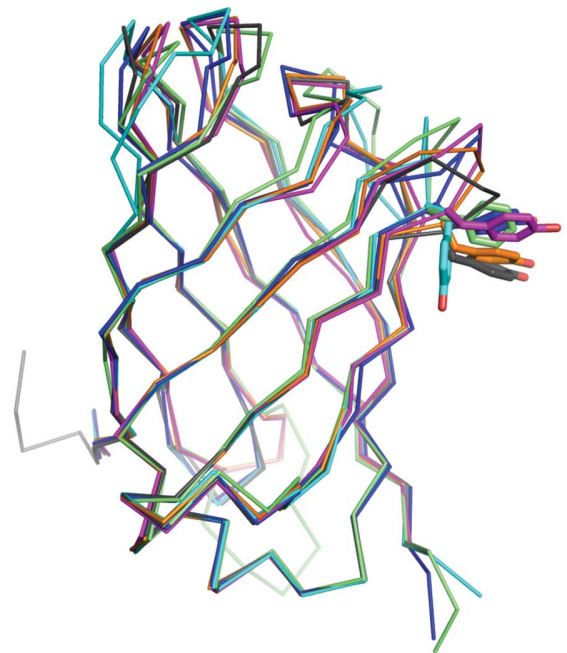

(c)

Figure 2

Overlay of R. equi Vap structures. (a) The previously published VapB structure with PDB code 4cv7 is coloured blue to red from the N-terminus to the C-terminus in order to illustrate the complex topology of the Vap $\beta$-barrel. (b) Overlay of three VapB structures: PDB entry 4cv7 in grey, PDB entry $7 \mathrm{~b} 1 \mathrm{z}$ chain $A$ in cyan and PDB entry 7b1z chain $B$ in blue. (c) Overlay of all published structures of $R$. equi Vaps: VapB, PDB entry 4cv7, grey; VapB, PDB entry 7b1z, chain $A$, cyan; VapB, PDB entry 7b1z, chain $B$, blue; VapD, PDB entry 4csb, lime; VapG, PDB entry 5aeo, chain $A$, orange; VapG, PDB entry 5 aeo, chain $B$, magenta. 
$\beta 5$ and $\beta 6$ and is very well defined in the electron density (Fig. 1b). The same three residues (Asn152, Asn159 and Asn161) from both chains contribute to nitrate binding. The position and orientation of the nitrate relative to the protein is similar but not identical in chains $A$ and $B$. The conformation of the asparagine side chains also differs between chains $A$ and $B$, particularly for Asn159, which coordinates a nitrate $\mathrm{O}$ atom via its amide $\mathrm{NH}_{2}$ in chain $A$, while in chain $B$ it coordinates the positively charged nitrate $\mathrm{N}$ atom via its amide $\mathrm{O}$ atom. It

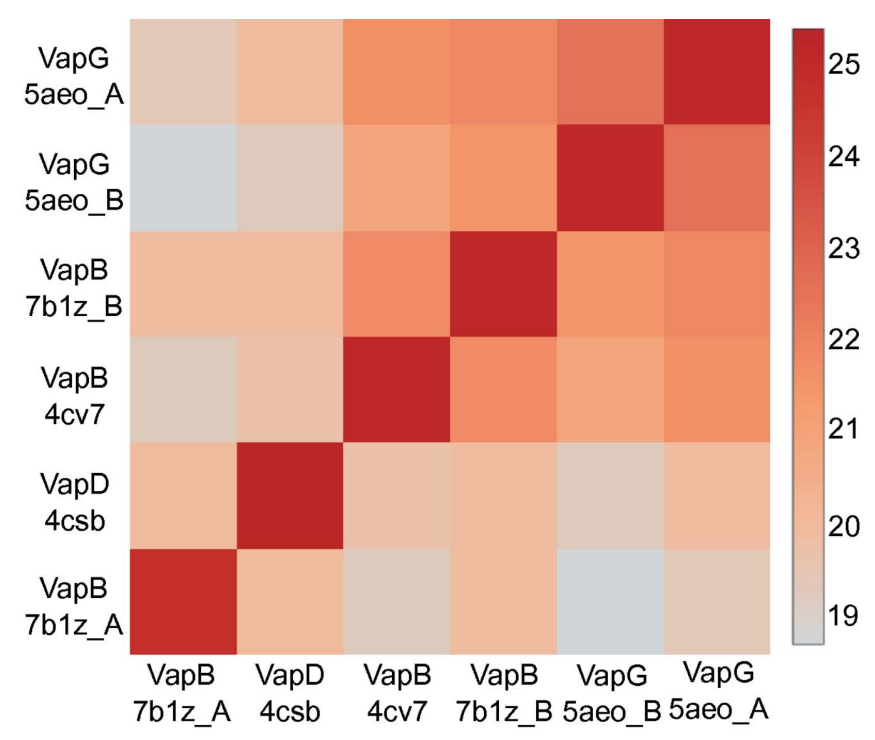

(a)

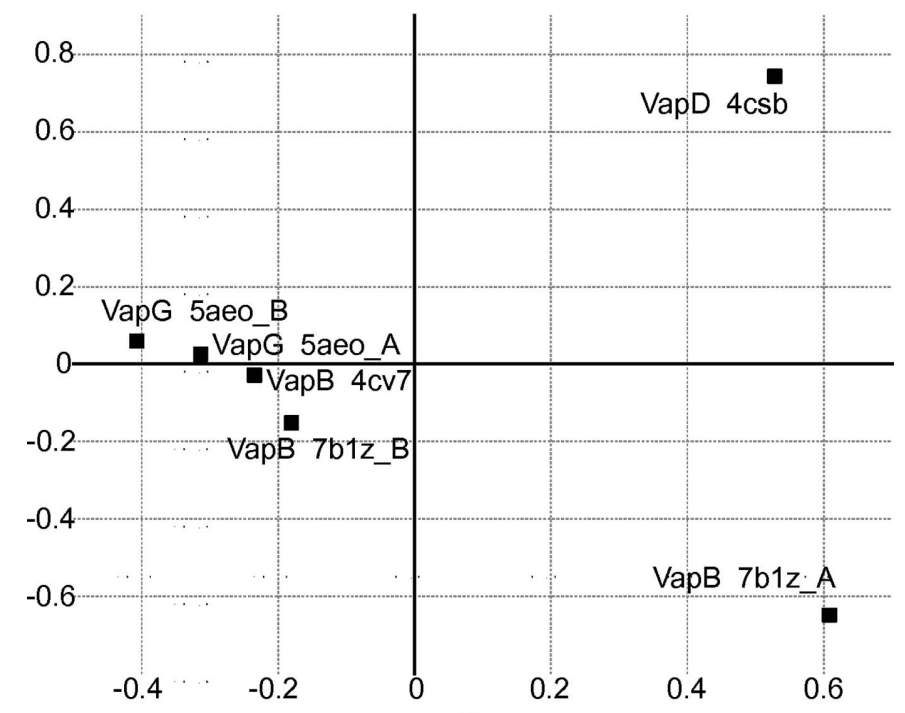

(b)

Figure 3

Structural comparison of loop regions at the top of the Vap $\beta$-barrel. An all-against-all analysis was performed with the $D A L I$ server (Holm, 2020) for truncated Vap structures, in which flexible ( $\mathrm{N}$ - and C-termini) or variable ( $\beta 2-\beta 3$ loop) residues had been removed. These six truncated Vap structures correspond to VapB residues 89-108 and 113-192. (a) Heat map of pairwise $Z$-scores. The bar on the right assigns colours to $Z$-scores. The pairwise root-mean-square distances (r.m.s.d.s) are given in Supplementary Table S1. (b) VapD and the open VapB structure appear as outliers in correspondence analysis, a multidimensional scaling method that positions data points with the most similar structural neighbourhoods near each other. The horizontal and vertical axes represent the first and the second eigenvector, respectively. appears unlikely that the bound nitrate has any biological relevance. Likewise, we consider the dimeric arrangement to be a mere crystal-packing contact for several reasons: (i) it does not have $C_{2}$ point-group symmetry as the vast majority of biological homodimers do (Schulz, 2010), (ii) it is not predicted to be stable in solution by the PISA server (Krissinel \& Henrick, 2007), (iii) it is not present in any of the other Vap structures, even when considering all crystallographic symmetry, and (iv) VapG behaves as monomer in solution, as shown by multi-angle laser light scattering (Whittingham et al., 2014).

An overlay of all three VapB monomers reveals that the bottom of the barrel is structurally largely invariant, while the loops at the top are flexible (Figs. $2 a$ and $2 b$ ). An extended comparison of all available Vap structures, including VapD and VapG (Fig. 2c), shows that the structural conservation of the bottom end extends across the Vap family, with the exception of the $\beta 2-\beta 3$ loop, which differs in conformation between VapB and VapG and harbours a five-residue insertion in VapD. The conformation of the loops at the top of the barrel apparently strongly depends on the crystal-packing environment. The loops at the top of the barrel are involved in crystal-packing contacts that differ between the three crystallographically independent VapB molecules (Supplementary Fig. S1). An all-against-all analysis of the available Vap structures using the $D A L I$ server (Holm, 2020) revealed that with regard to these loops, chain $A$ of our new VapB structure is most distant from the other Vap structures. Two of the three VapB structures (PDB entries 4cv7 and 7b1z chain $B$ ) cluster with the two VapG structures (PDB entries 5aeo chain $A$ and 5 aeo chain $B$ ). VapD (PDB entry 4csb) and VapB (PDB entry $7 \mathrm{~b} 1 \mathrm{z}$ chain $A$ ) are distinct from the VapG/VapB cluster and from each other (Fig. 3). The side chain of Tyr157 also adopts a unique rotamer in PDB entry $7 \mathrm{~b} 1 \mathrm{z}$ chain $A$ (Fig. 2). In the first VapB structure the loops at the top of the barrel were suggested to be flexible because of their high $B$ factors. Moreover, the $\beta 1-\beta 2$ and the $\beta 7-\beta 8$ loops were modelled with

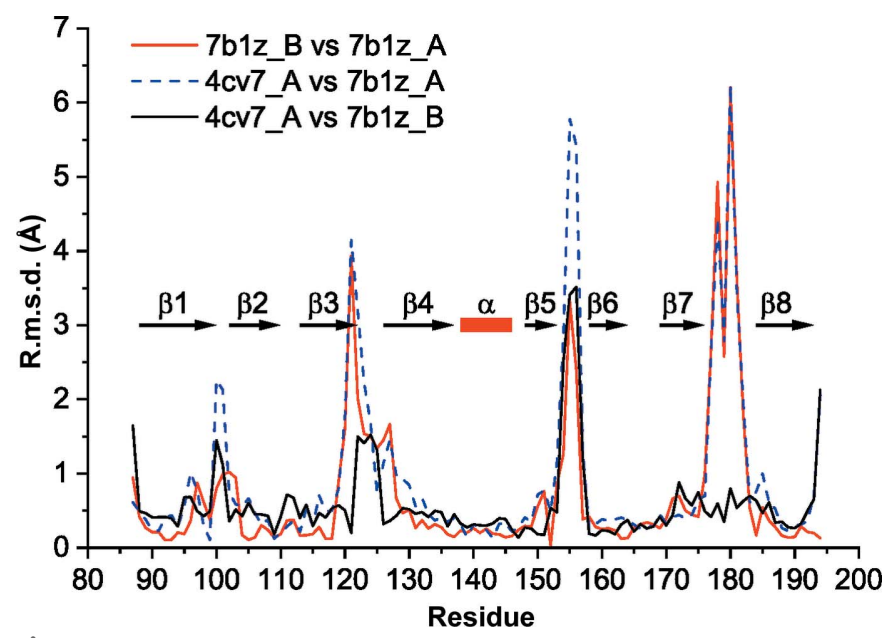

Figure 4

Pairwise comparison of all VapB structures. The per-residue r.m.s.d. of $\mathrm{C}^{\alpha}$ atoms was calculated with $L S Q K A B$ (Kabsch, 1976). The position of secondary-structure elements is indicated for the first VapB structure (PDB entry 4cv7). 
double conformations (Geerds et al., 2014). A pairwise comparison of all three VapB structures shows that the largest backbone displacements of chain $A$ of our new structure (PDB entry $7 \mathrm{~b} 1 \mathrm{z}$ chain $A$ ) compared with the first VapB structure (PDB entry $4 \mathrm{cv} 7$ ) or chain $B$ of the new VapB structure (PDB entry $7 \mathrm{~b} 1 \mathrm{z}$ chain $B$ ) occur in the $\beta 7-\beta 8$ loop, the $\beta 5-\beta 6$ loop and the $\beta 3-\beta 4$ loop (Fig. 4). In contrast, the $\beta 7-\beta 8$ loop has almost identical conformations in PDB entries $4 \mathrm{cv} 7$ and $7 \mathrm{~b} 1 \mathrm{z}$ chain $B$. This is reminiscent of streptavidin, in which loops connecting the eight $\beta$-strands and especially the flexible binding loop can adopt different conformations either depending on the state (unbound versus biotin-bound) or as a consequence of crystal-packing interactions (Freitag et al., 1997; Le Trong et al., 2011). Compared with the other Vap structures, the $\beta 3-\beta 4$ loop and the $\beta 7-\beta 8$ loop of PDB entry $7 \mathrm{~b} 1 \mathrm{z}$ chain $A$ move outwards in opposite directions, creating a pocket between them. Okoko and coworkers suggested that Vap proteins may have functions that only manifest upon the conformational changes that frequently accompany the binding of small molecules to proteins (Okoko et al., 2015). Our new VapB structure reveals that despite the crossover connections, VapB can form a large cavity between the $\beta 3-\beta 4$ loop and the $\beta 7-\beta 8$ loop (Fig. 5). In all other Vap structures smaller cavities are present between the $\beta 5-\beta 6$ loop and the
VapB

PDB entry $7 \mathrm{~b} 1 \mathrm{z}$ chain $A$

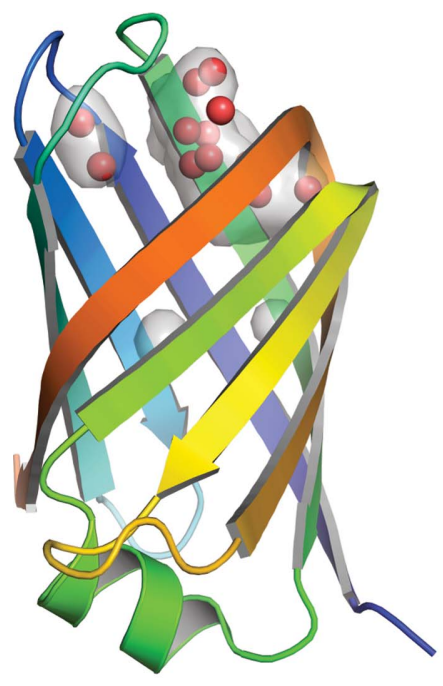

VapD

PDB entry $4 \mathrm{csb}$

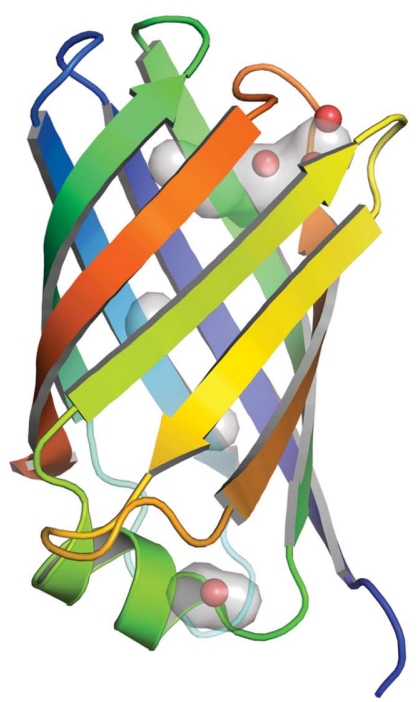

VapB

PDB entry $7 \mathrm{~b} 1 \mathrm{z}$ chain $B$

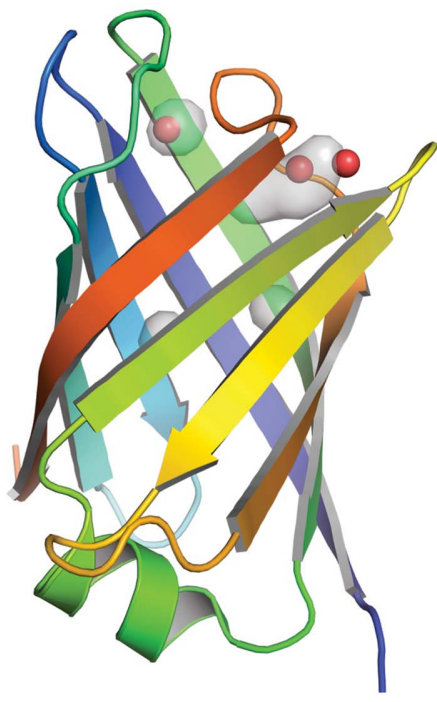

VapG
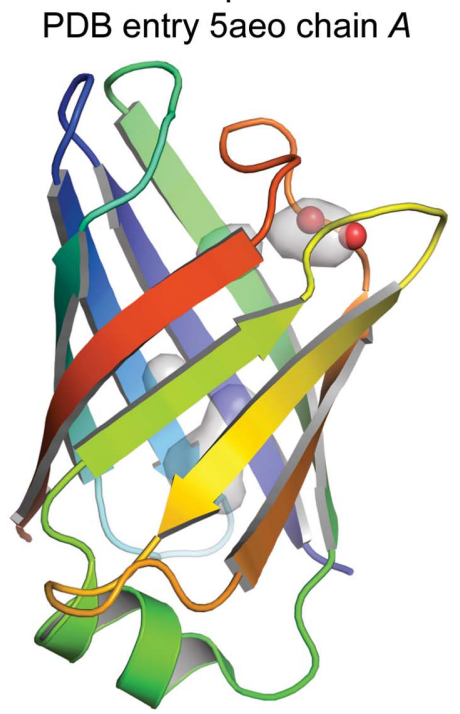

VapB PDB entry 4cv7

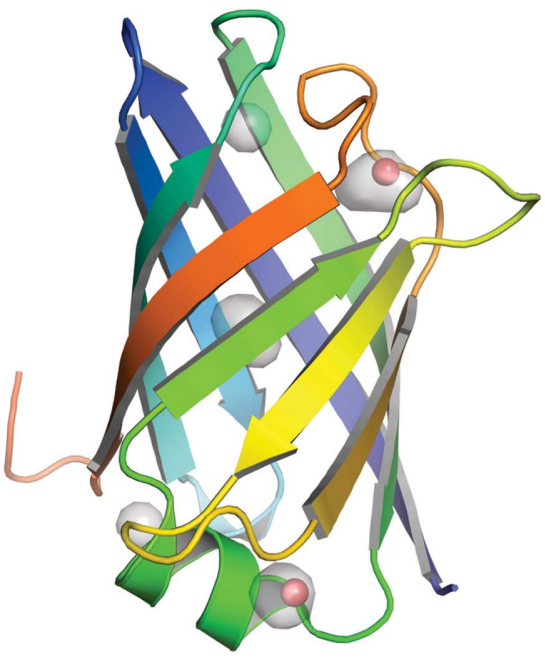

VapG

PDB entry 5aeo chain $B$

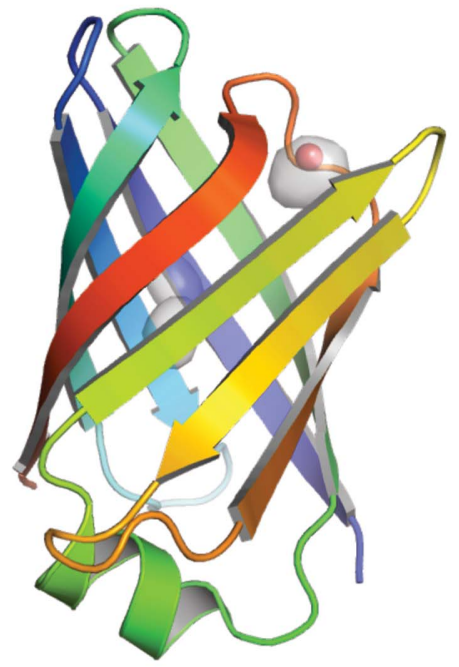

Figure 5

Cavities in Vap structures. All Vaps were structurally aligned. The cartoon representation is coloured blue to red from the N-terminus to the C-terminus. The setting 'Cavities and Pockets Only' in the surface representation of $P y M O L$ was used to display completely enclosed and surface-accessible cavities as a transparent grey surface. Water molecules located completely or partially within these pockets are shown as red spheres. Only chain $A$ of the new VapB structure (PDB entry $7 \mathrm{~b} 1 \mathrm{z}$ chain $A$ ) has a large, water-filled pocket between the $\beta 3-\beta 4$ loop and the $\beta 7-\beta 8$ loop. 


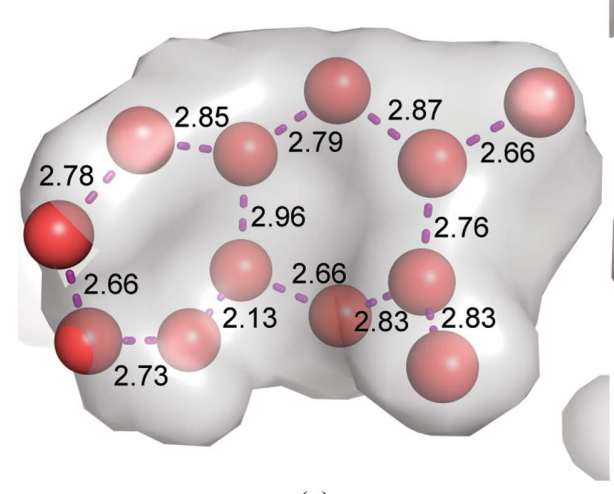

(a)

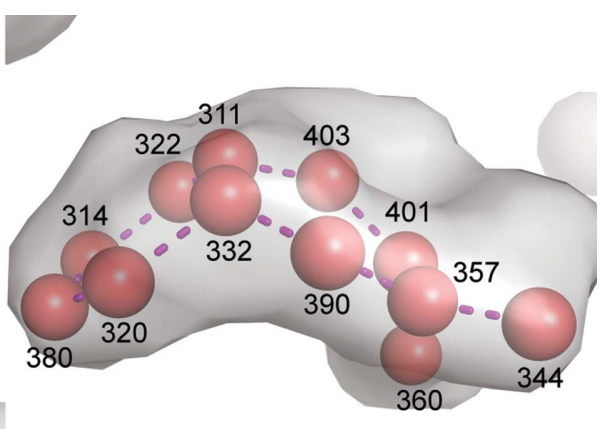

(b)

Figure 6

Water molecules within the large VapB pocket. The two views are rotated by $90^{\circ}$ relative to each other. (a) Distances given in $\AA$ are shown as purple dashed lines. (b) The residue numbering in PDB entry $7 \mathrm{~b} 1 \mathrm{z}$ chain $A$ is indicated in black. Waters 311 and 322 , which are only $2.13 \AA$ apart, are modelled with partial occupancies of 0.5 .

$\beta 7-\beta 8$ loop. The bottom of the large VapB cavity is formed by hydrophobic residues (Va196, Phe105, Phe130, Ala153, Leu158 and Ala177), while its side and mouth contain both hydrophobic (for example Ile124 and Val181) and polar (for example Ser98, Gln103, Thr123, Tyr151 and Ser179) residues. The large cavity in VapB contains 12 ordered water molecules (A311, A314, A320, A322, A332, A344, A357, A360, A380, A390, A401 and A403), giving a hint on the size and shape of a putative ligand (Fig. 6). An overlay of VapB and bradavidin 2 in complex with biotin (PDB entry 4ggz; Leppiniemi et al., 2013) using the TopMatch server (Wiederstein \& Sippl, 2020) places the biotin-binding site of bradavidin 2 in roughly the same position as the cavity of VapB (Supplementary Fig. S2).

In summary, our new VapB structure suggests that the helixcapped bottom of the Vap $\beta$-barrel is structurally highly conserved and very rigid, whereas the top end is flexible. A conformational change of the loops at the top end of the VapB barrel opens a cavity that might act as binding site for a ligand. Uncovering the identity of a putative Vap ligand would substantially advance our understanding of how Vaps exert their virulence function during $R$. equi infections.

\section{Acknowledgements}

The synchrotron macromolecular crystallography data were collected on beamline P14 operated by EMBL Hamburg at the PETRA III storage ring, DESY, Hamburg, Germany. We would like to thank Dr Thomas Schneider and Dr Gleb Bourenkov for assistance in using the beamline. Open access funding enabled and organized by Projekt DEAL.

\section{Funding information}

AH was supported by grant HA 1929/14-1 from the Deutsche Forschungsgemeinschaft.

\section{References}

Barden, S., Schomburg, B., Conradi, J., Backert, S., Sewald, N. \& Niemann, H. H. (2014). Acta Cryst. D70, 1391-1400. von Bargen, K., Scraba, M., Krämer, I., Ketterer, M., Nehls, C., Krokowski, S., Repnik, U., Wittlich, M., Maaser, A., Zapka, P., Bunge, M., Schlesinger, M., Huth, G., Klees, A., Hansen, P., Jeschke, A., Bendas, G., Utermöhlen, O., Griffiths, G., Gutsmann, T., Wohlmann, J. \& Haas, A. (2019). Cell. Microbiol. 21, e12958.

Casañal, A., Lohkamp, B. \& Emsley, P. (2020). Protein Sci. 29, 1069 1078.

Diederichs, K., McSweeney, S. \& Ravelli, R. B. G. (2003). Acta Cryst. D59, 903-909.

Fernandez-Mora, E., Polidori, M., Lührmann, A., Schaible, U. E. \& Haas, A. (2005). Traffic, 6, 635-653.

Freitag, S., Le Trong, I., Klumb, L., Stayton, P. S. \& Stenkamp, R. E. (1997). Protein Sci. 6, 1157-1166.

Geerds, C., Wohlmann, J., Haas, A. \& Niemann, H. H. (2014). Acta Cryst. F70, 866-871.

Holm, L. (2020). Protein Sci. 29, 128-140.

Jain, S., Bloom, B. R. \& Hondalus, M. K. (2003). Mol. Microbiol. 50, $115-128$.

Kabsch, W. (1976). Acta Cryst. A32, 922-923.

Kabsch, W. (2010). Acta Cryst. D66, 125-132.

Krissinel, E. \& Henrick, K. (2007). J. Mol. Biol. 372, 774-797.

Leppiniemi, J., Meir, A., Kähkönen, N., Kukkurainen, S., Määttä, J. A., Ojanen, M., Jänis, J., Kulomaa, M. S., Livnah, O. \& Hytönen, V. P. (2013). Protein Sci. 22, 980-994.

Letek, M., Ocampo-Sosa, A. A., Sanders, M., Fogarty, U., Buckley, T., Leadon, D. P., González, P., Scortti, M., Meijer, W. G., Parkhill, J., Bentley, S. \& Vázquez-Boland, J. A. (2008). J. Bacteriol. 190, 57975805.

Le Trong, I., Wang, Z., Hyre, D. E., Lybrand, T. P., Stayton, P. S. \& Stenkamp, R. E. (2011). Acta Cryst. D67, 813-821.

Liebschner, D., Afonine, P. V., Baker, M. L., Bunkóczi, G., Chen, V. B., Croll, T. I., Hintze, B., Hung, L.-W., Jain, S., McCoy, A. J., Moriarty, N. W., Oeffner, R. D., Poon, B. K., Prisant, M. G., Read, R. J., Richardson, J. S., Richardson, D. C., Sammito, M. D., Sobolev, O. V., Stockwell, D. H., Terwilliger, T. C., Urzhumtsev, A. G., Videau, L. L., Williams, C. J. \& Adams, P. D. (2019). Acta Cryst. D75, 861-877.

Livnah, O., Bayer, E. A., Wilchek, M. \& Sussman, J. L. (1993). Proc. Natl Acad. Sci. USA, 90, 5076-5080.

McCoy, A. J., Grosse-Kunstleve, R. W., Adams, P. D., Winn, M. D., Storoni, L. C. \& Read, R. J. (2007). J. Appl. Cryst. 40, 658-674.

Meyer, T., Zumbrägel, N., Geerds, C., Gröger, H. \& Niemann, H. H. (2020). Biomolecules, 10, 1130.

Moritzer, A. \& Niemann, H. H. (2019). Protein Sci. 28, 2112-2118. 
Murshudov, G. N., Skubák, P., Lebedev, A. A., Pannu, N. S., Steiner, R. A., Nicholls, R. A., Winn, M. D., Long, F. \& Vagin, A. A. (2011). Acta Cryst. D67, 355-367.

Ng, J. T., Dekker, C., Reardon, P. \& von Delft, F. (2016). Acta Cryst. D72, 224-235.

Niemann, H. H., Schmoldt, H., Wentzel, A., Kolmar, H. \& Heinz, D. W. (2006). J. Mol. Biol. 356, 1-8.

Okoko, T., Blagova, E. V., Whittingham, J. L., Dover, L. G. \& Wilkinson, A. J. (2015). Vet. Microbiol. 179, 42-52.

Ribeiro, M. G., Takai, S., Guazzelli, A., Lara, G. H. B., da Silva, A. V., Fernandes, M. C., Condas, L. A. Z., Siqueira, A. K. \& Salerno, T. (2011). Res. Vet. Sci. 91, 478-481.

Schreiner, M. \& Niemann, H. H. (2012). BMC Struct. Biol. 12, 13.

Schulz, G. E. (2010). J. Mol. Biol. 395, 834-843.

Takai, S., Sekizaki, T., Ozawa, T., Sugawara, T., Watanabe, Y. \& Tsubaki, S. (1991). Infect. Immun. 59, 4056-4060.

Thoms, S., Hofhuis, J., Thöing, C., Gärtner, J. \& Niemann, H. H. (2011). J. Struct. Biol. 175, 362-371.
Tkachuk-Saad, O. \& Prescott, J. (1991). J. Clin. Microbiol. 29, 26962700.

Vázquez-Boland, J. A. \& Meijer, W. G. (2019). Mol. Microbiol. 112,115.

Weber, P. C., Ohlendorf, D. H., Wendoloski, J. J. \& Salemme, F. R. (1989). Science, 243, 85-88.

Whittingham, J. L., Blagova, E. V., Finn, C. E., Luo, H., MirandaCasoLuengo, R., Turkenburg, J. P., Leech, A. P., Walton, P. H., Murzin, A. G., Meijer, W. G. \& Wilkinson, A. J. (2014). Acta Cryst. D70, 2139-2151.

Wiederstein, M. \& Sippl, M. J. (2020). Nucleic Acids Res. 48, W31W35.

Willingham-Lane, J. M., Coulson, G. B. \& Hondalus, M. K. (2018). PLoS One, 13, e0204475.

Wright, L. M., Carpinone, E. M., Bennett, T. L., Hondalus, M. K. \& Starai, V. J. (2018). Mol. Microbiol. 107, 428-444.

Zink, M. C., Yager, J. A., Prescott, J. F. \& Fernando, M. A. (1987). Vet. Microbiol. 14, 295-305. 